\title{
Effects of extremely low frequency pulsed magnetic fields on diabetic nephropathy in streptozotocin-treated rats
}

\author{
Feijiang Li ${ }^{\dagger}$, Tao Lei ${ }^{\dagger}$, Kangning Xie ${ }^{\dagger}$, Xiaoming Wu, Chi Tang, Maogang Jiang, Juan Liu, Erping Luo ${ }^{*}$ \\ and Guanghao Shen ${ }^{*}$
}

\section{${ }^{*}$ Correspondence:} erpingluo@hotmail.com; shenguanghao2002@sina. com

${ }^{\dagger}$ Feijiang Li, Tao Lei and Kangning Xie contributed equally to this work School of Biomedical Engineering, Fourth Military Medical University, Xi'an, China

\begin{abstract}
Background: Extremely low frequency pulsed magnetic fields (ELFPMF) have been shown to induce Faraday currents and measurable effects on biological systems. A kind of very high frequency electromagnetic field was reported that it improved the symptoms of diabetic nephropathy (DN) which is a major complication of diabetes. However, few studies have examined the effects of ELFPMF DN at the present. The present study was designed to investigate the effects of ELFPMF on DN in streptozotocin (STZ)-induced type 1 diabetic rats.
\end{abstract}

Methods: Adult male SD rats were randomly divided into three weight-matched groups: Control (non-diabetic rats without DN), DN + ELFPMF (diabetic rats with DN exposed to ELFPMF, 8 h/days, 6 weeks) and DN (diabetic rats with DN exposed to sham ELFPMF). Renal morphology was examined by light and electron microscopy, vascular endothelial growth factor (VEGF)-A and connective tissue growth factor (CTGF) were measured by enzyme linked immune sorbent assay.

Results: After 6 weeks'ELFPMF exposure, alterations of hyperglycemia and weight loss in STZ-treated rats with DN were not found, while both positive and negative effects of ELFPMF on the development of DN in diabetic rats were observed. The positive one was that ELFPMF exposure attenuated the pathological alterations in renal structure observed in STZ-treated rats with DN, which were demonstrated by slighter glomerular and tubule-interstitial lesions examined by light microscopy and slighter damage to glomerular basement membrane and podocyte foot processes examined by electron microscopy. And then, the negative one was that ELFPMF stimulation statistically significantly decreased renal expression of VEGF-A and statistically significantly increased renal expression of CTGF in diabetic rats with $D N$, which might partially aggravate the symptoms of DN.

Conclusion: Both positive and negative effects of ELFPMF on the development of DN in diabetic rats were observed. The positive effect induced by ELFPMF might play a dominant role in the procession of DN in diabetic rats, and it is suggested that the positive effect should be derived from the correction of pathogenic diabetes-induced mediators.

Keywords: Pulsed magnetic fields, Diabetic nephropathy, Streptozotocin, rats 


\section{Background}

Diabetic nephropathy (DN) is a major complication of diabetes [1]. Approximately $20-40 \%$ of patients with type 1 or type 2 diabetes mellitus develop DN [2]. DN is the leading cause of chronic kidney disease accounting for nearly $50 \%$ of all end-stage renal disease worldwide [3]. DN is characterized by increased glomerular permeability to proteins and excessive extracellular matrix accumulation in the mesangium, eventually resulting in glomerulosclerosis and progressive renal impairment [4].

The increasing prevalence of DN worldwide represents a major societal issue because of the enormous expense associated with kidney replacement therapy [5]. Current therapies that aim to lower blood glucose are not effective in blocking renal damage, and cotreatment with renoprotective drugs often results in potential toxicity, poor tolerability and ineffectiveness for some percent of diabetic patients [6]. Therefore, there is an urgent need to explore other non-pharmacological novel therapeutic modalities with efficacy and safety, particularly when patients with DN require a combined treatment with an oral renoprotective drug to preserve normal renal function and to prevent or slow the progression of DN.

Near infrared light is a kind of extremely high frequency electromagnetic wave, which has much higher frequency than ELFPMF. A study has reported that a near infrared light (670 nm low-level light) improved renal function and antioxidant defense capabilities in the kidney of streptozotocin (STZ)-induced type 1 diabetic rats [7]. Near infrared light and ELFPMF have very different effects in their interaction with living tissue, while ELFPMF is more widely studied than near infrared light in biological systems by our literature review. According to our literature review, ELFPMF has been shown to induce non-thermal effects on biological systems [8]. However, few studies have examined the effects of ELFPMF on DN at the present. Therefore, we aimed to investigate the effects of ELFPMF on kidney complications induced by diabetes.

In the current study, we used STZ-treated diabetic rats with DN to assess the effects of whole-body exposure to $15 \mathrm{~Hz}$ ELFPMF whose peak magnetic flux density (MFD) was approximate $1.6 \times 10^{-3} \mathrm{~T}$ on the development of renal changes in DN by using morphological examination and ELISA analysis for VEGF-A and CTGF. The ELFPMF was generated by a self-produced apparatus and the exposure duration was $8 \mathrm{~h}$ per day, 6 days a week for 6 weeks. Our results demonstrate that ELFPMF had two-sided effects on renal damage induced by diabetes.

\section{Methods}

\section{Experimental diabetes}

Thirty-two adult male Sprague-Dawley rats, weighting $300 \pm 20$ g, were provided by Animal Center of the Fourth Military Medical University and housed in a room (Animal Center of the Fourth Military Medical University, Xi'an, China) with controlled temperature $\left(23 \pm 1{ }^{\circ} \mathrm{C}\right)$, relative humidity $(50 \sim 60 \%)$, and alternately light-dark cycle $(12 \mathrm{~h} / 12 \mathrm{~h})$, with access to standard pellet and clean water. Eight rats were treated as non-diabetic control animals, and the rest 24 rats were used to induce diabetic models. Diabetes mellitus was induced by an intraperitoneal injection of STZ (55 mg/kg body weight) in freshly prepared citrate buffer ( $\mathrm{pH} 4.5$ ) after an overnight fast. Similarly, equivalent dose of sterile citrate buffer solution was injected into the control rats. 
Confirmation of hyperglycemia was made three days after STZ injection, and only STZ-treated rats whose glucose concentration of the tail venous blood measured by OneTouch SureStep Plus glucometer (Lifescan, Milpitas, CA, USA) were higher than $20 \mathrm{mmol} / \mathrm{L}$ were considered as qualified diabetic models [9]. Eight rats were excluded from the qualified diabetic models because of low blood glucose levels. The rest of rats were randomized into three weight-matched groups (eight in each group): non-diabetic rats without DN control group (Control), diabetic rats with DN exposed to ELFPMF group (DN + ELFPMF) which were subjected to whole-body exposure to $15 \mathrm{~Hz}$ ELFPMF $8 \mathrm{~h}$ (09:00-17:00) everyday, 6 days a week for 6 weeks and diabetic rats with DN exposed to sham ELFPMF exposure group (DN). Although the same ELFPMF apparatus was employed in DN group, the ELFPMF stimulator was not activated. ELFPMF stimulation was carried out the next day after confirmation of hyperglycemia. The current study was performed in adherence to the National Institutes of Health guidelines for the use of experimental animals, and all animal protocols were approved by the Committee for Ethical Use of Experimental Animals of the Fourth Military Medical University.

\section{ELFPMF apparatus}

The ELFPMF exposure system (GHY-III, FMMU, Xian, China; China Patent no.ZL02224739.4) was composed of a pulsed signal generator (Fig. 1b) and a modified Helmholtz coils assembled with three-coil array. This apparatus has presented has been described in detail in our previous study [10].

The modified Helmholtz coils was composed of three identical coils which were in series connection, with the same coil diameters of $800 \mathrm{~mm}$ and placed coaxially with a distance of $304 \mathrm{~mm}$ apart. Each coil was made up of enameled coated copper wire

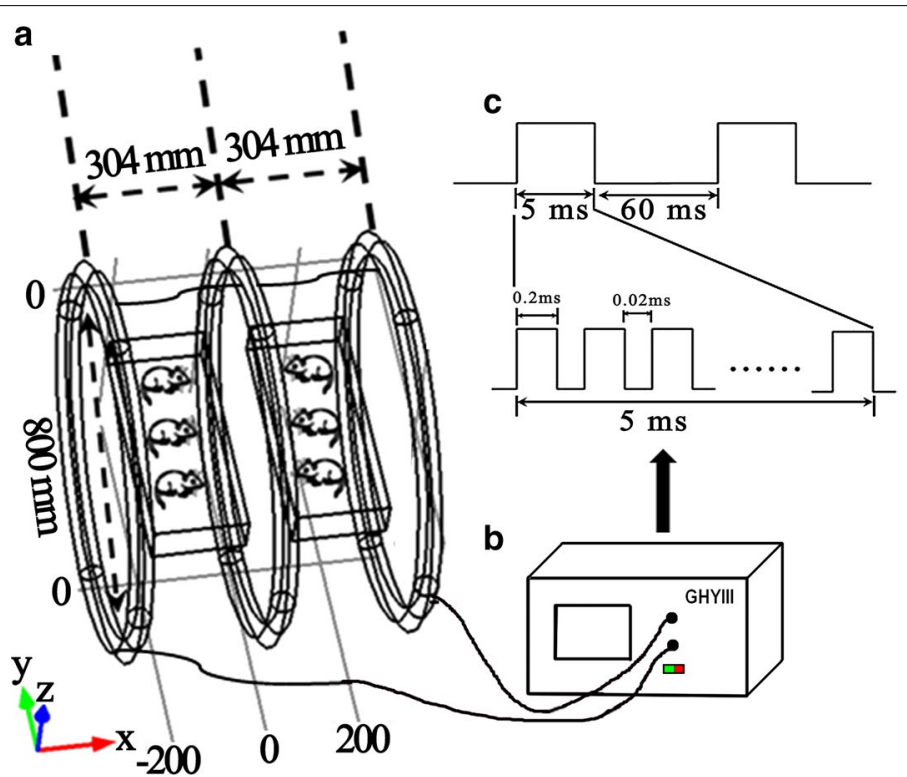

Fig. 1 Schematic drawing of the ELFPMF apparatus and pulse protocol. a Modified Helmholtz coils assembled with three-coil array. b The pulsed signal generator GHY-III. c The open-circuit voltage waveform generated by GHY- III with a repetitive burst frequency at $15 \mathrm{~Hz}$ (burst width, $5 \mathrm{~ms}$; burst wait, $60 \mathrm{~ms}$; pulse width, $0.2 \mathrm{~ms}$; pulse wait, $0.02 \mathrm{~ms}$; pulse rise and fall time: $0.3,2.0 \mu \mathrm{s}$ ) 
with $0.8 \mathrm{~mm}$ diameter. The number of turns of the central coil was 266 , and the number of turns of the two outside coils was 500 (Fig. 1a). The modified Helmholtz configured with these parameters has presented significantly upgraded uniformity of MFD [10]. As depicted in Fig. 1c, the pulsed signal apparatus generated an open circuit waveform which was composed of a pulsed burst (burst width, $5 \mathrm{~ms}$; burst wait, $60 \mathrm{~ms}$; pulse width, $0.2 \mathrm{~ms}$; pulse wait, $0.02 \mathrm{~ms}$; pulse rise and fall time: 0.3, $2.0 \mu \mathrm{s}$ ) repeated at $15 \mathrm{~Hz}$. This type of waveform has been proved effective in the prevention and treatment of bone disorders, cardiovascular and neurological diseases by our research group over a long period of time [10-12].

Two cubic plastic rat cages containing rats in DN + ELFPMF group were put in the center of every two neighboring coils (the length of the cage was along $\mathrm{O}-\mathrm{Y}$ direction) and cages were supported by stands to let the activities of rats restrict on the XY plane which had higher intensity and better uniformity of MFD (Fig. 1). The distribution of the peak MFD was measured by using a Gaussmeter (Model 455 DMP Gaussmeter, Lake Shore Cryotronics, USA), and the measurement result was $(1.6 \pm 0.1) \times 10^{-3} \mathrm{~T}$ $($ mean $\pm \mathrm{SD}$ ) in the exposure area (cage: $50 \mathrm{~cm}$ long, $20 \mathrm{~cm}$ wide and $15 \mathrm{~cm}$ high).

\section{Measurements of weights and blood glucose levels}

The weights and blood glucose levels of all rats were evaluated prior to STZ administration, and there were no statistically significant differences for these parameters among three groups. In addition, weights and blood glucose levels of all rats were measured after 6-week ELFPMF exposure period.

\section{Get the tissue samples}

Six weeks after ELFPMF stimulation in STZ-treated rats with DN, all rats were anesthetized by an intraperitoneal injection of $7 \%$ chloral hydrate solution $(0.45 \mathrm{ml} / 100 \mathrm{~g})$ prior to collecting the kidney. Both kidneys for each rat were harvested and blotted. Left or right kidney for each rat was randomly chosen to carry morphological examination and ELISA analysis respectively.

\section{Light microscopy of renal morphology}

The renal cortex samples were fixed by immersion in $10 \%$ neutral buffered formalin. After being embedded in paraffin, $4 \mu \mathrm{m}$ sections were stained with hematoxylin-eosin (HE), periodic acid Schiff (PAS), and Masson's trichrome. Two sections for every staining method were got per rat, and five different sites of renal glomeruli were viewed per section. All the slides were examined by a renal pathologist (YW) in single blind fashion.

\section{Ultrastructural examination of renal glomeruli}

The renal cortex was dissected and post fixed by immersion in the fixative solution ( $2 \%$ paraformaldehyde, $2 \%$ glutaraldehyde, $0.1 \mathrm{M}$ cacodylate buffer at $\mathrm{pH} 7.3$ ) for $2 \mathrm{~h}$ at $4{ }^{\circ} \mathrm{C}$, and washed in $0.1 \mathrm{M}$ cacodylate buffer, and osmicated for $4 \mathrm{~h}$ in $1 \% \mathrm{OsO}_{4}$ (Fluka). Sections of renal cortex were rinsed in $0.1 \mathrm{M}$ cacodylate buffer, dehydrated and embedded in epoxy 812-Araldite (Polysciences). Ultra-thin sections $(80 \mathrm{~nm})$ were subsequently cut, collected on cellodincoated single slot grids and stained with uranyl acetate and lead citrate. The microphotographs were obtained using a transmission electron microscope 
(JEM-1230, Japan) operated at $80 \mathrm{keV}$, and the thickness of glomerular basement membrane (GBM) was measured using the ruler function of the software. Average GBM thickness for each rat was calculated from 30 measurements obtained from different sites of GBM, and eight rats were chosen to evaluate the thickness of GBM for each group.

\section{ELISA}

After cutting kidney cortex samples, we checked the weight, added PBS (pH 7.2-7.4), rapidly frozen with liquid nitrogen, maintained samples at $2-8{ }^{\circ} \mathrm{C}$ after melting, added PBS ( $\mathrm{pH}$ 7.4), homogenized by grinders, carried centrifugation 20-min at the speed of 2000-3000 r.p.m. and finally removed the supernatant.

Assays were performed in 48-well immunoplates (Chemicon International). ELISA analyses for VEGF-A and CTGF were performed using commercially available kits for rat VEGF-A (Yonghui Biotechnology Co., Ltd, Beijing, China) and rat CTGF (Yonghui Biotechnology Co., Ltd, Beijing, China) respectively. Experiments were performed according to the manufacturers' instructions [13].

\section{Statistics}

Statistical analyses were carried out using SPSS (version 14.0, SPSS, IL, USA). All values were expressed as mean \pm SD. $P<0.05$ was considered statistically significant. All data sets were independent, normally distributed, and with equal variance. Comparisons between multiple groups were performed by one-way ANOVA followed by Bonferroni's post hoc test.

\section{Results}

\section{Body weights and whole blood glucose levels}

After 6-week diabetic period, as shown in Fig. 2, One-way ANOVA with Bonferroni's post hoc determined that the mean body weight of DN group and DN + ELFPMF group were statistically significantly lower than that in the Control group $(P<0.05)$. After STZ injection, rats consistently lost weight. Although there was slightly less loss of the body weight in ELFPMF treated diabetic rats, no significant difference between DN + ELFPMF group and DN group was found $(P>0.05)$. Six weeks' ELFPMF stimulation did not statistically significantly affect the loss of body weight caused by diabetes. Similarly, after 6-week diabetic period, one-way ANOVA with Bonferroni's post hoc revealed that the average blood glucose levels of DN group and DN + ELFPMF group were statistically significantly higher than that in the Control group $(P<0.05)$ (Fig. 3). STZ administration caused a rapid elevation of average blood glucose levels $(>27.8 \mathrm{mmol} / \mathrm{L})$ within one week, which persisted for up to 6 weeks. Although the blood glucose levels were slightly lower in ELFPMF treated diabetic rats, there was no significant difference between DN + ELFPMF group and DN group $(P>0.05)$. Six weeks' ELFPMF stimulation did not statistically significantly affect the hyperglycemia caused by diabetes. 


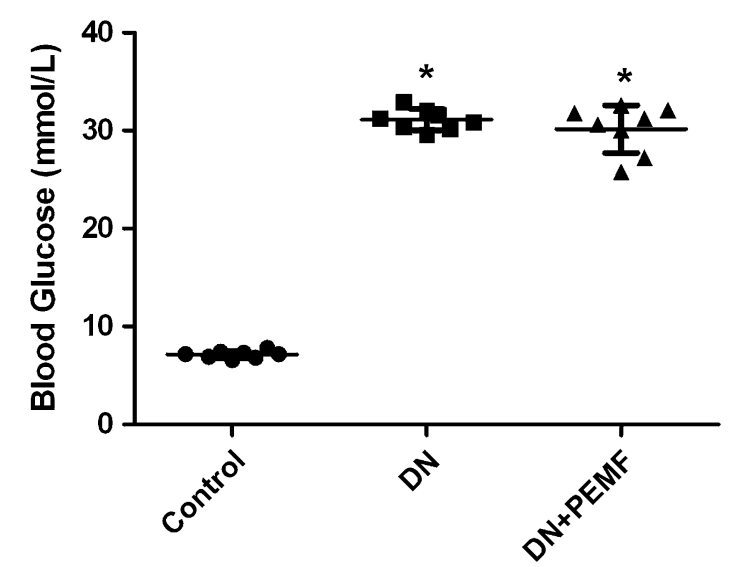

Fig. 2 Scatter plot for weights of STZ-induced diabetic rats with DN after 6-week exposure to ELFPMF. Plot is expressed as mean $\pm S D(n=8)$, ${ }^{*} P<0.05$ vs Control group

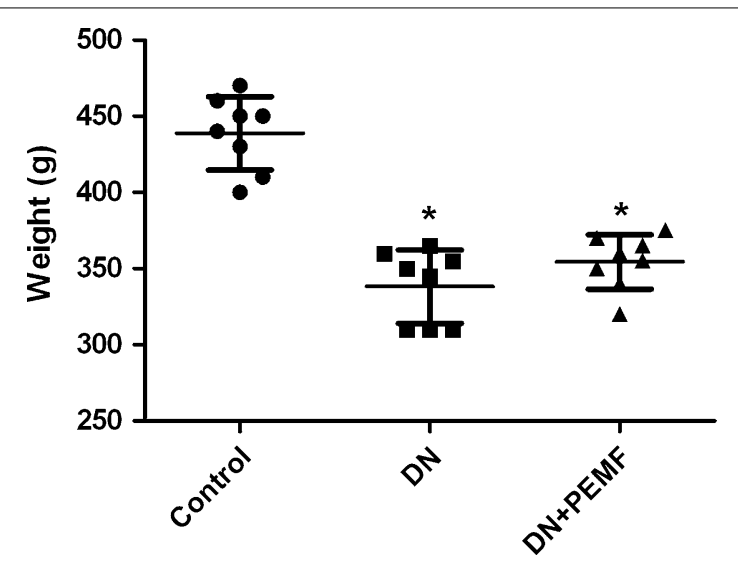

Fig. 3 Scatter plot for blood glucose levels of STZ-induced diabetic rats with DN after 6-week exposure to ELFPMF. Plot is expressed as mean $\pm \mathrm{SD}(\mathrm{n}=8)$, ${ }^{*} P<0.05$ vs Control group

\section{Light microscopy}

Glomerular lesions were observed in HE-stained tissue sections from STZ-injected rats at the end of the 6-week experimental period (Fig. 4, HE panel). Glomerular hypertrophy and mesangial matrix expansion were observed in the DN group at the end of the 6-week experiment period. However, these changes were ameliorated in the DN + ELFPMF group compared with DN group. Treatment with ELFPMF partially restored the normal morphology of glomeruli in diabetic rats (Fig. 4, HE panel).

Glycogen deposition in the glomeruli was measured by PAS staining to indicate the severity of glomerulosclerosis. PAS staining showed STZ-induced glomerulosclerosis, which was partially improved in ELFPMF treated diabetic rats (Fig. 4, PAS panel).

Since extracellular matrix deposition is another hallmark of glomerulosclerosis, collagen was visualized using Masson's trichrome staining (Fig. 4, Trichrome panel). STZ treatment resulted in collagen accumulation inside glomeruli or in the periglomerular area, which was reduced in ELFPMF treated group. 


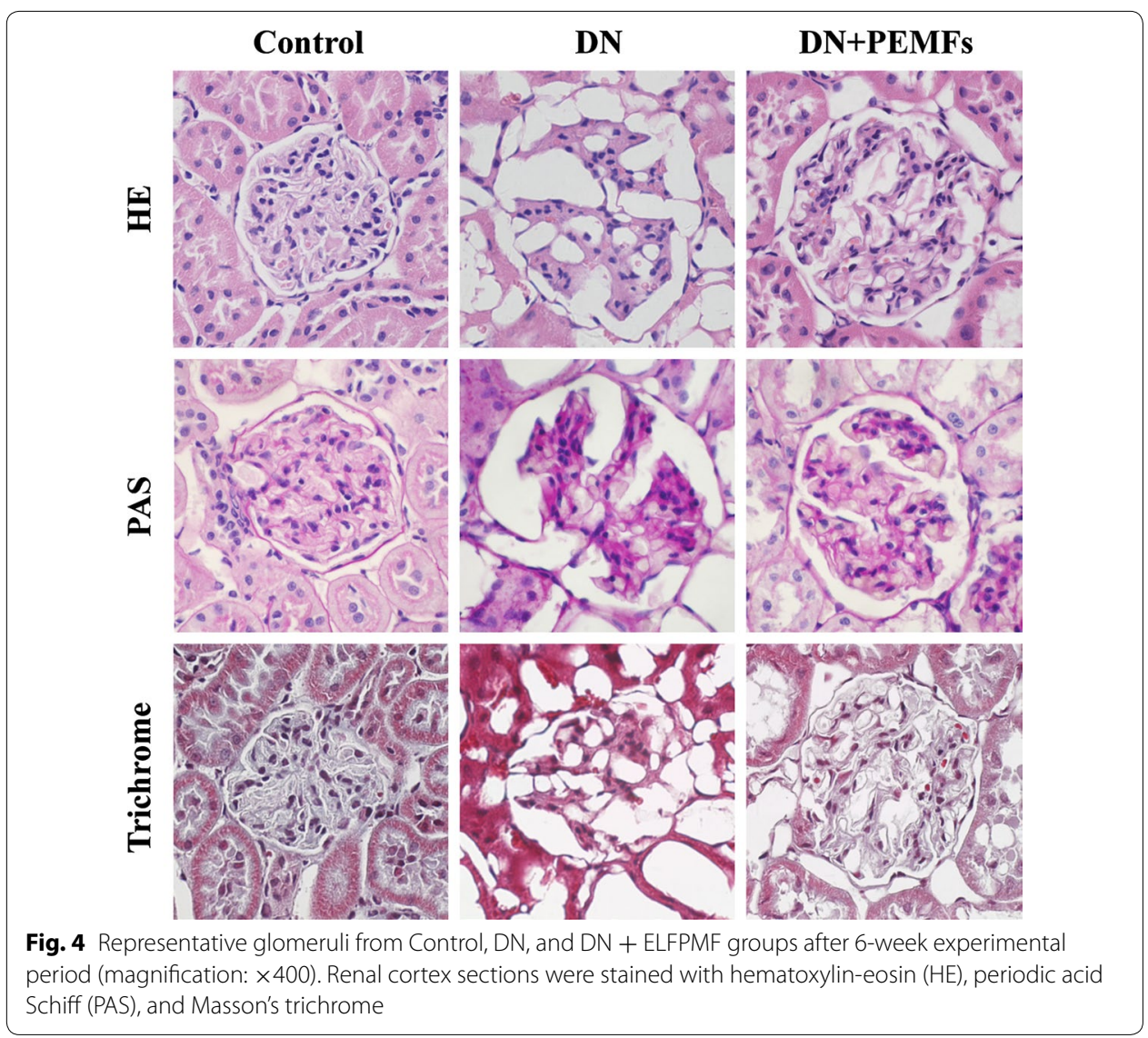

These results clearly demonstrate that these diabetic rats develop glomerular hypertrophy, glomerular extracellular matrix accumulation, increasement of GBM thickness and mesangial proliferation within 6 weeks after diabetes debut. What's more, the results from our study also revealed the ability of ELFPMF treatment to attenuate STZ-induced pathological alterations in renal structure during the progression of DN.

\section{Electron microscopy}

Ultrastructural examination of glomeruli was obtained by using transmission electron microscopy after 6-week experimental period in all rats. In Control group, glomeruli with normal structure and morphology were observed and podocyte foot processes were regularly shaped (Fig. 5a). Glomeruli from diabetic rats displayed some degree of thickness of the GBM which has been an independent criterion for glomerulosclerosis (Fig. 5b, arrow, d). What's more, effacement and confluence of podocyte foot processes were observed in DN group (Fig. 5b). In DN + ELFPMF group, glomeruli and podocyte foot processes were abnormal, but the damage was slighter than that in the DN group (Fig. 5c). Especially, the thickness of GBM was improved by 6-week ELFPMF exposure (Fig. 5d). 


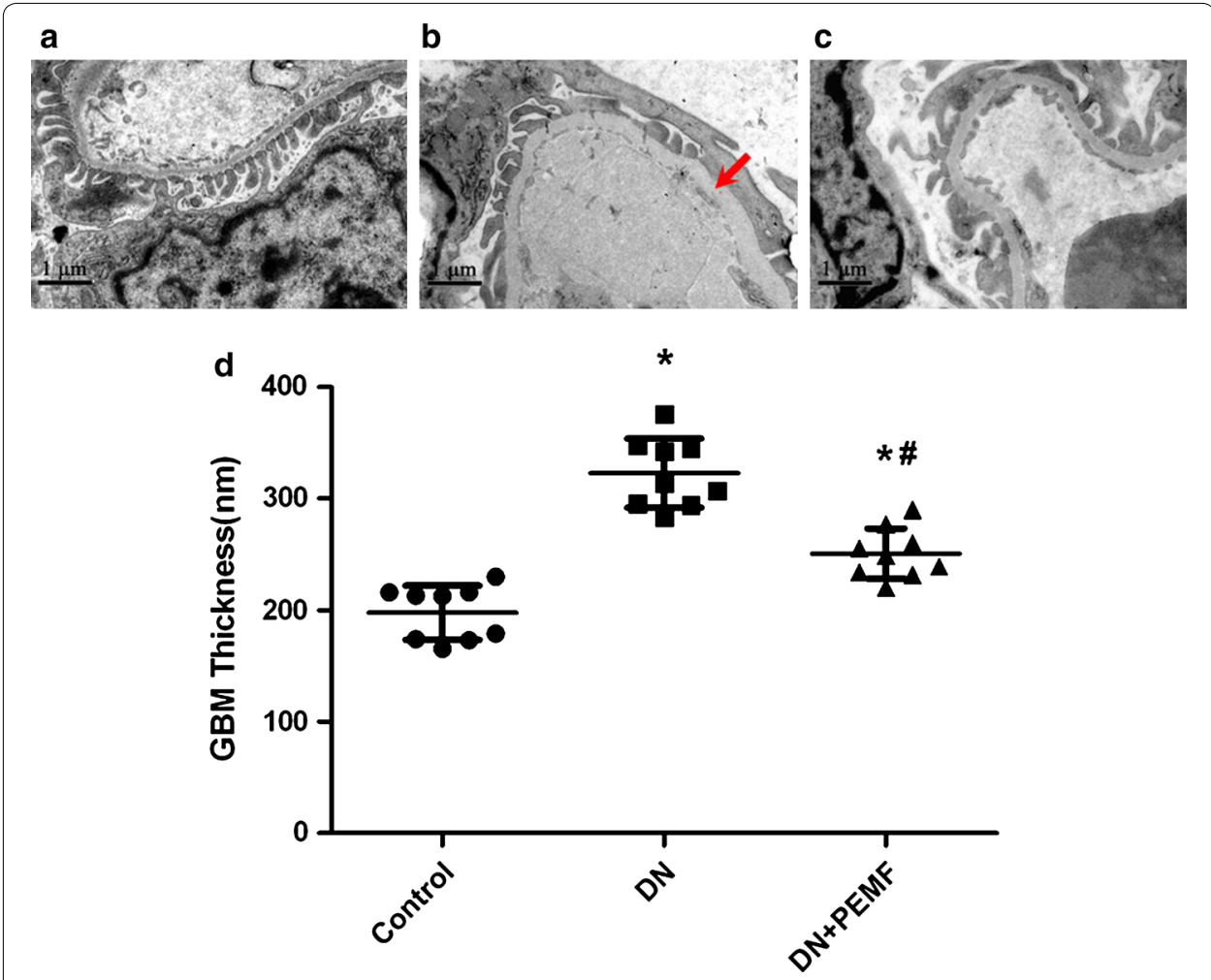

Fig. 5 Electron microscopy of the GBM and podocyte foot processes in Control, DN, and DN + ELFPMF groups after 6-week experimental period are displayed (magnification: $\times 10,000$ ). a Control group: glomeruli with normal structure and morphology were observed and podocyte foot processes were regularly shaped. b DN group: Glomeruli from diabetic rats displayed some degree of thickness of the GBM, effacement and confluence of podocyte foot processes were also observed. c DN + ELFPMF group: glomeruli and podocyte foot processes were abnormal, but the damage was slighter than that in DN group. d Scatter plot for GBM thickness in Control, DN, and DN + ELFPMF groups are plotted as mean $\pm S D(n=8$ per group). The mean value of GBM thickness measured at 30 different sites was considered as the GBM per rat. ${ }^{*} \mathrm{P}<0.05$ vs Control group, ${ }^{\#} P<0.05$ vs DN group

\section{Effects of ELFPMF on renal expression of VEGF-A}

Bonferroni-adjusted pairwise comparisons revealed that the renal expression of VEGFA in DN group and DN + ELFPMF group were statistically significantly lower than that in the Control group $(P<0.01)$ (Fig. 6). The renal expression of VEGF-A significantly decreased in diabetic rats compared to that in the non-diabetic rats. In addition, the renal expression of VEGF-A in DN + ELFPMF group was statistically significantly lower than that in the DN group $(P<0.05)$ (Fig. 6). ELFPMF stimulation significantly decreased the renal expression of VEGF-A in diabetic rats.

\section{Effects of ELFPMF on renal expression of CTGF}

Bonferroni-adjusted pairwise comparisons revealed that the renal expression of CTGF in DN group and DN + ELFPMF group were statistically significantly higher than that in the Control group $(P<0.01)$ (Fig. 7). The renal expression of CTGF significantly increased in the diabetic rats compared to that in the non-diabetic rats. In addition, the renal expression of CTGF in DN + ELFPMF group was statistically significantly higher 


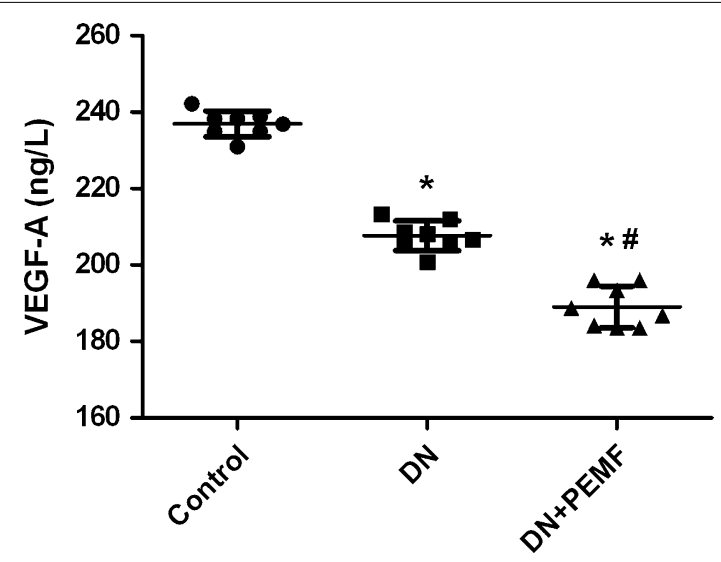

Fig. 6 ELISA analysis for VEGF-A expression in renal cortex in Control, DN, and DN + ELFPMF groups after 6-week experimental period $(n=8) .{ }^{*} P<0.05$ vs Control group; ${ }^{P} P<0.05$ vs DM group

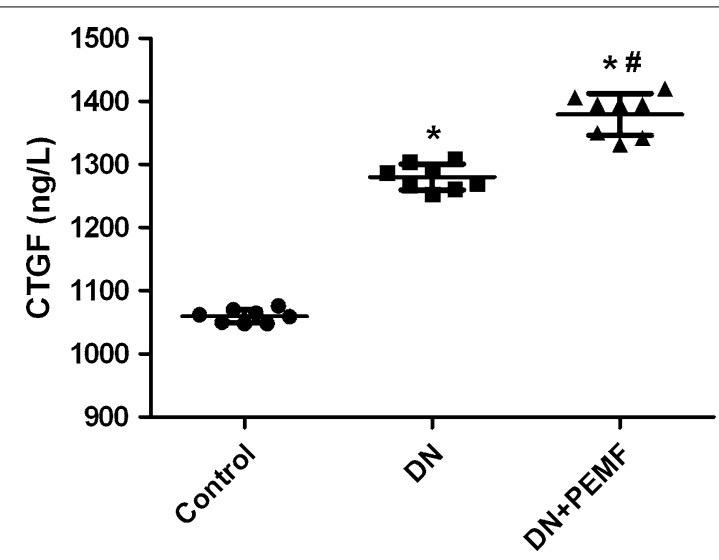

Fig. 7 ELISA analysis for CTGF expression in renal cortex in Control, DN, and DN + ELFPMF groups after 6-week experimental period $(n=8) .{ }^{*} P<0.05$ vs Control group; ${ }^{P} P<0.05$ vs DM group

than that in the DN group $(P<0.05)$ (Fig. 7). ELFPMF stimulation significantly increased the renal expression of CTGF in diabetic rats.

\section{Discussion}

In the current investigation, a marked decrease in body weight of diabetic rats was observed 6 weeks after the debut of diabetes as compared to that in non-diabetic control rats. The reduction in body weight is probably related to the osmotic diuresis and dehydration induced by diabetic hyperglycemia [14]. Meanwhile, blood glucose level rose immediately after the STZ injection, reached quite a high level at first week $(>27.8 \mathrm{mmol} / \mathrm{L})$, and then remained approximately at $27 \mathrm{mmol} / \mathrm{L}$. The results of the current study have confirmed previous findings that blood glucose level elevated and body weight decreased in diabetic rats after STZ administration [15]. Our results also revealed that ELFPMF stimulation did not statistically significantly prevent the weight loss caused by diabetes, which is consistent to our and others' previous investigation [10, 14]. However, contrary to the findings researched by Mert [14] and Jing [11] who observed that 
ELFPMF had efficacy in anti-hyperglycemia in diabetic rats, we found that the application of ELFPMF did not statistically significantly alter hyperglycemia in diabetic rats during the whole experimental period ( 6 weeks). The different effects of ELFPMF on hyperglycemia might be ascribed to the different standards for diabetic model adopted by us and them. The higher standards $(>20 \mathrm{mmol} / \mathrm{L}$ ) for diabetic model adopted by us than Mert's and Jing's ( $>16.7 \mathrm{mmol} / \mathrm{L}$ ) might cause a severe damage which could not be reversed by ELFPMF.

Obviously, a prerequisite for a clear understanding of the pathophysiological mechanisms of nephropathic appearance and treatment is to know if there are definite structural changes in the kidney and to what extent they exist in the patients or experimental animal models. The pathology of DN is characterized by increased glomerular permeability to proteins and excessive extracellular matrix accumulation in the mesangium, eventually resulting in glomerulosclerosis and progressive renal impairment [3, 4]. In the present study, some evidences of pathological alterations of the glomerulus such as progressive glomerular hypertrophy, mesangial matrix expansion, thickened GBM and effacement and confluence of podocyte foot processes were observed in STZ-treated rats with DN. Similar results were also reported by other investigators [16, 17]. In addition to this, our morphological study of kidney revealed that long-term ELFPMF stimulation partially prevented or slowed the progression of DN in STZ-treated rats, which appears to be seldom reported in animal models for $\mathrm{DN}$ by other investigators.

Our findings confirm earlier studies on kidney biopsies from diabetes patients and other glomerular diseases in which VEGF-A expression was also decreased in the glomeruli [18]. Similarly, another study also reported that glomerular expression of VEGF-A was reduced 1 week after STZ induction [19]. It is more likely that the downregulation of VEGF-A, results from a reduction of insulin secretion caused by streptozotocin in different disease stages [20]. What's more, VEGF-A is normally produced by podocytes, and podocyte loss observed by ultrastructural examination of glomeruli in our study might also cause down-regulation of VEGF-A [21, 22]. However, this would seem to contradict an experimental study on diabetic rats in which VEGF-A mRNA levels in the cortex and glomeruli were increased [23]. The reason for the differences between our findings and those reported in the literature may be that the shorter duration of disease studied than theirs [24]. In addition to this, our results also revealed that ELFPMF stimulation significantly decreased the renal expression of VEGF-A in diabetic rats, and the down-regulation of VEGF-A induced by ELFPMF is similarly reported by our previous study and other investigators [10,12].

Glomerular hypertrophy and accumulation of extracellular matrix components, which is mediated predominantly by the prosclerotic cytokines TGF- $\beta$ and CTGF, are other characteristic features of DN [4]. In our study, after 6 weeks of STZ-induced diabetes, there was a significant increase in expression of CTGF in rat renal cortex. Our data on expression of CTGF in renal cortex are in agreement with previously studies which reported that overexpression of CTGF at the mRNA or protein level has previously been demonstrated in vivo in experimental diabetic rats [25] and human kidney with DN [26], as well as in cultured high glucose stimulated HK-2 cells [27]. What's more, our results also revealed that 6-week administration of ELFPMF statistically significantly increased the expression of CTGF measured by ELISA. The effects of ELFPMF on expression of 
CTGF in our study are similar to other investigations which reported that ELFPMF could promote extracellular matrix production and mineralization in osteoblastlike cells [28] and accelerate extracellular matrix synthesis in connective tissue [29].

In our study, the down-regulation of VEGF-A and up-regulation of CTGF induced by 6-week ELFPMF exposure in renal cortex indicate that ELFPMF might aggravate the symptoms of DN. However, actually, 6-week exposure to ELFPMF stimulation partially prevented the development of glomeruli degeneration in STZ-treated rats with DN by using morphological examinations. Therefore, we hypothesized that although the regulation of VEGF-A and CTGF induced by ELFPMF might partially aggravate the development of DN, ELFPMF might ameliorate kidney complications induced by diabetes through other mechanisms, because the pathogenesis of DN is complex and involves multiple pathways that lead to kidney injury, including the polyol pathway [30], protein kinase $\mathrm{C}$ [31], advanced glycation end products [32], high glucose-induced generation of reactive oxygen species (ROS) [33], and proinflammatory cytokines. These diabetes-induced mediators interact in their adverse effects on the kidney. In addition, suppression of ROS production [34] and anti-inflammatory [35, 36] effects have been found by numerous studies. Therefore, it is not surprising that correction of any of them by ELFPMF results in amelioration of DN. Therefore, we would argue that long-term ELFPMF stimulation might ameliorate kidney complications in diabetic rats by correction of pathogenic diabetes-induced mediators, which might lead to slighter glomerular and tubulointerstitial lesions compared to those of the DN group observed in the present study.

\section{Conclusions}

It is suggested that ELFPMF might have two kinds of effects on diabetic rats with DN. One is negative effect, which is demonstrated by statistically significantly decreased renal expression of VEGF-A and significantly increased renal expression of CTGF in diabetic rats, and the other is positive effect, which is inferred from the slighter pathological alterations in renal structure in DN + ELFPMF group than that in DN group. More importantly, the positive effect induced by ELFPMF might play a dominant role in the procession of DN in diabetic rats, and this positive effect induced by ELFPMF might result from correction of pathogenic diabetes-induced mediators. However, further research is required to elucidate the specific mechanisms for the two-sided effects of ELFPMF on DN.

\section{Authors' contributions}

FL, TL and KX carried out the animal experiment, participated in the sequence alignment and drafted the manuscript. CT and XW built the ELFPMF device. MJ participated in The design of the study and performed the statistical analysis. Juan Liu carried out Tissue staining work. GS and EL conceived of the study, and participated in its design and coordination and helped to draft the manuscript. All authors read and approved the final manuscript.

\footnotetext{
Acknowledgements technical assistance.

Competing interests

The authors declare that they have no competing interests.

Received: 13 September 2015 Accepted: 22 December 2015

Published online: 19 January 2016
}

The authors would like to thank Tao Chen (Department of Clinical Aerospace Medicine, Fourth Military Medical University), Xiangmin Yang (Department of Ophthalmology, Xijing Hospital, Fourth Military Medical University) and Jingmei Huang (Department of General Dentistry, Stomatological Hospital, Fourth Military Medical University) for their excellent 


\section{References}

1. Caramori ML, Kim Y, Huang C, Fish AJ, Rich SS, Miller ME, Russell G, Mauer M. Cellular basis of diabetic nephropathy: 1. Study design and renal structural-functional relationships in patients with long-standing type 1 diabetes. Diabetes. 2002;51(2):506-13.

2. Finne P, Reunanen A, Stenman S, Groop PH, Gronhagen-Riska C. Incidence of end-stage renal disease in patients with type 1 diabetes. JAMA. 2005;294(14):1782-7.

3. Dronavalli S, Duka I, Bakris GL. The pathogenesis of diabetic nephropathy. Nat Clin Pract Endocrinol Metab. 2008;4(8):444-52.

4. Molitch ME, DeFronzo RA, Franz MJ, Keane WF, Mogensen CE, Parving HH, Steffes MW. Nephropathy in diabetes Diabetes Care. 2004;27(Suppl 1):S79-83.

5. Decleves AE, Sharma K. New pharmacological treatments for improving renal outcomes in diabetes. Nat Rev Nephrol. 2010;6(6):371-80.

6. Zheng H, Whitman SA, Wu W, Wondrak GT, Wong PK, Fang D, Zhang DD. Therapeutic potential of Nrf2 activators in streptozotocin-induced diabetic nephropathy. Diabetes. 2011;60(11):3055-66.

7. Lim J, Sanders RA, Snyder AC, Eells JT, Henshel DS, Watkins JR. Effects of low-level light therapy on streptozotocininduced diabetic kidney. J Photochem Photobiol B. 2010;99(2):105-10.

8. Markov MS, Colbert AP. Magnetic and electromagnetic field therapy. J Back Musculoskelet Rehabil. 2000;15(1):17-29.

9. Samii A, Unger J, Lange W. Vascular endothelial growth factor expression in peripheral nerves and dorsal root ganglia in diabetic neuropathy in rats. Neurosci Lett. 1999;262(3):159-62.

10. Lei T, Jing D, Xie K, Jiang M, Li F, Cai J, Wu X, Tang C, Xu Q, Liu J, et al. Therapeutic effects of 15 Hz pulsed electromagnetic field on diabetic peripheral neuropathy in streptozotocin-treated rats. PLoS One. 2013;8(4):e61414

11. Jing D, Cai J, Shen G, Huang J, Li F, Li J, Lu L, Luo E, Xu Q. The preventive effects of pulsed electromagnetic fields on diabetic bone loss in streptozotocin-treated rats. Osteoporos Int. 2011;22(6):1885-95.

12. Luo E, Shen G, Xie K, Wu X, Xu Q, Lu L, Jing X. Alimentary hyperlipemia of rabbits is affected by exposure to lowintensity pulsed magnetic fields. Bioelectromagnetics. 2007;28(8):608-14.

13. McArthur K, Feng B, Wu Y, Chen S, Chakrabarti S. MicroRNA-200b regulates vascular endothelial growth factormediated alterations in diabetic retinopathy. Diabetes. 2011;60(4):1314-23.

14. Mert T, Gunay I, Ocal I. Neurobiological effects of pulsed magnetic field on diabetes-induced neuropathy. Bioelectromagnetics. 2010;31(1):39-47.

15. Hoybergs YM, Biermans RL, Meert TF. The impact of bodyweight and body condition on behavioral testing for painful diabetic neuropathy in the streptozotocin rat model. Neurosci Lett. 2008;436(1):13-8.

16. Chen HY, Huang XR, Wang W, Li JH, Heuchel RL, Chung AC, Lan HY. The protective role of Smad7 in diabetic kidney disease: mechanism and therapeutic potential. Diabetes. 2011;60(2):590-601.

17. Matsushita Y, Ogawa D, Wada J, Yamamoto N, Shikata K, Sato C, Tachibana H, Toyota N, Makino H. Activation of peroxisome proliferator-activated receptor delta inhibits streptozotocin-induced diabetic nephropathy through anti-inflammatory mechanisms in mice. Diabetes. 2011;60(3):960-8.

18. Bailey E, Bottomley MJ, Westwell S, Pringle JH, Furness PN, Feehally J, Brenchley PE, Harper SJ. Vascular endothelial growth factor mRNA expression in minimal change, membranous, and diabetic nephropathy demonstrated by non-isotopic in situ hybridisation. J Clin Pathol. 1999;52(10):735-8.

19. Singh AK, Gudehithlu KP, Pegoraro AA, Singh GK, Basheerudin K, Robey RB, Arruda JA, Dunea G. Vascular factors altered in glucose-treated mesangial cells and diabetic glomeruli. Changes in vascular factors impair endothelial cell growth and matrix. Lab Invest. 2004;84(5):597-606.

20. Hale LJ, Hurcombe J, Lay A, Santamaria B, Valverde AM, Saleem MA, Mathieson PW, Welsh GI, Coward RJ. Insulin directly stimulates VEGF-A production in the glomerular podocyte. Am J Physiol Renal Physiol. 2013;305(2):F182-8

21. Eyre J, Burton JO, Saleem MA, Mathieson PW, Topham PS, Brunskill NJ. Monocyte- and endothelial-derived microparticles induce an inflammatory phenotype in human podocytes. Nephron Exp Nephrol. 2011;119(3):e58-66.

22. Lee EY, Chung $\mathrm{CH}$, Kim JH, Joung HJ, Hong SY. Antioxidants ameliorate the expression of vascular endothelial growth factor mediated by protein kinase C in diabetic podocytes. Nephrol Dial Transplant. 2006;21(6):1496-503.

23. Cooper ME, Vranes D, Youssef S, Stacker SA, Cox AJ, Rizkalla B, Casley DJ, Bach LA, Kelly DJ, Gilbert RE. Increased renal expression of vascular endothelial growth factor (VEGF) and its receptor VEGFR-2 in experimental diabetes. Diabetes. 1999:48(11):2229-39.

24. Baelde HJ, Eikmans M, Lappin DW, Doran PP, Hohenadel D, Brinkkoetter PT, van der Woude FJ, Waldherr R, Rabelink TJ, de Heer E, et al. Reduction of VEGF-A and CTGF expression in diabetic nephropathy is associated with podocyte loss. Kidney Int. 2007;71(7):637-45.

25. Lai PB, Zhang L, Yang LY. Quercetin ameliorates diabetic nephropathy by reducing the expressions of transforming growth factor-beta1 and connective tissue growth factor in streptozotocin-induced diabetic rats. Ren Fail. 2012;34(1):83-7.

26. Adler SG, Kang SW, Feld S, Cha DR, Barba L, Striker L, Striker G, Riser BL, LaPage J, Nast CC. Glomerular mRNAs in human type 1 diabetes: biochemical evidence for microalbuminuria as a manifestation of diabetic nephropathy. Kidney Int. 2001;60(6):2330-6.

27. Li GS, Jiang WL, Yue XD, Qu GW, Tian JW, Wu J, Fu FH. Effect of astilbin on experimental diabetic nephropathy in vivo and in vitro. Planta Med. 2009:75(14):1470-5.

28. Heermeier K, Spanner M, Trager J, Gradinger R, Strauss PG, Kraus W, Schmidt J. Effects of extremely low frequency electromagnetic field (EMF) on collagen type I mRNA expression and extracellular matrix synthesis of human osteoblastic cells. Bioelectromagnetics. 1998;19(4):222-31.

29. Aaron RK, Ciombor DM. Therapeutic effects of electromagnetic fields in the stimulation of connective tissue repair. Cell Biochem. 1993;52(1):42-6.

30. Dunlop M. Aldose reductase and the role of the polyol pathway in diabetic nephropathy. Kidney Int Suppl. 2000:77:S3-12. 
31. Koya D, Jirousek MR, Lin YW, Ishii H, Kuboki K, King GL. Characterization of protein kinase C beta isoform activation on the gene expression of transforming growth factor-beta, extracellular matrix components, and prostanoids in the glomeruli of diabetic rats. J Clin Invest. 1997;100(1):115-26.

32. Yang CW, Vlassara $H$, Peten EP, He CJ, Striker GE, Striker LJ. Advanced glycation end products up-regulate gene expression found in diabetic glomerular disease. Proc Natl Acad Sci USA. 1994;91(20):9436-40.

33. Brownlee M. The pathobiology of diabetic complications: a unifying mechanism. Diabetes. 2005;54(6):1615-25.

34. Lim S, Kim SC, Kim JY. Protective Effect of 10-Hz, 1-mT Electromagnetic Field Exposure Against Hypoxia/Reoxygenation Injury in HK-2 Cells. Biomed Environ Sci. 2015;28(3):231-4.

35. Costin GE, Birlea SA, Norris DA. Trends in wound repair: cellular and molecular basis of regenerative therapy using electromagnetic fields. Curr Mol Med. 2012;12(1):14-26.

36. Kaszuba-Zwoinska J, Ciecko-Michalska I, Madroszkiewicz D, Mach T, Slodowska-Hajduk Z, Rokita E, Zaraska W, Thor P. Magnetic field anti-inflammatory effects in Crohn's disease depends upon viability and cytokine profile of the immune competent cells. J Physiol Pharmacol. 2008;59(1):177-87.

Submit your next manuscript to BioMed Central and we will help you at every step:

- We accept pre-submission inquiries

- Our selector tool helps you to find the most relevant journal

- We provide round the clock customer support

- Convenient online submission

- Thorough peer review

- Inclusion in PubMed and all major indexing services

- Maximum visibility for your research

Submit your manuscript at 